noch anführen, dass im ersten Octoberhefte des Dinglerschen Journals ein kurzes Referat über Untersuchungen gegeben ist, welche J. M. Ma isch „über das Verhalten des Chloroforms gegen das Licht" ausgeführt hat. Das angewandte Chloroform hatte ein spec. Gew. von 1,492 bei $21^{\circ} \mathrm{C}$., war absolut frei von Săure und färbte Schwefelsäure nicht. Das Resultat dieser Untersuchungen war folgendes :

1) Reines Chloroform von spec. Gew. 1,492 muss bei seiner Aufbewahrung vor dem Zutritte von Licht gänzlich geschiitzt sein.

2) Um Chloroform bei Lichtzutritt aufbewahren zu können, ohne dass es sich zersetzt, muss sein spec. Gew. verringert werden, was am besten durch Zusatz von éiwa 2 Drachm. 95proc. Alkohol zu $1 \mathrm{Pfd}$. Chloroform von 1,4970 spec. Gew. geschieht. Chloroform durch Alkoholzusatz auf das spec. Gew. 1,475 gebracht, soll sich selbst bei Gegenwart von Wasser durch directes Sonnenlicht nicht zersetzen.

\title{
Zur Milchprüfung;
}

von

Dr. Julius Erdmann.

Die Unzuverlässigkeit des bisher in Hannover und mehren andern Städten Deutschlands hauptsächlich zur Voruntersuchung der Milch angewandten Instrumentes, des Galactometers, hat mir Veranlassung gegeben, eine Reihe von Milchuntersuchungen vorzunehmen, welche vorzugsweise bezweckten, die Brauchbarkeit eines anderen Instruments, des Galactoskops von $\mathrm{Vogel}$, namentlich zur Voruntersuchung der Milch*) festzustellen, und wird es, glaube ich, für meine Fachgenossen von Interesse sein, die Resultate dieser Prüfungen, so wie die ander-

*) Zur endgültigen Untersuchung für die Behörden gewährt jedenfalls die Bestimmung sämmtlicher festen Bestandtheile grössere Sicherheit. 
weitig bei dieser Gelegenheit gesammelten Erfahrungen in einem kurzen Berichte niedergelegt zu sehen. Das Vogel'sche Instrument ist zwar schon von verschiedenen Seiten geprüft, aber von den betreffenden Autoren von andern Gesichtspuncten aus kritisirt worden.

\section{Versuche mit Normalmilch.}

$\mathrm{Zu}$ den optischen Proben wurden 100 Cubikcentimeter Wasser so lange mit Milch versetzt, bis eine 5 Millimeter dicke Schicht des Gemisches die Conturen eines vom Galactoskop 12 Zoll entfernten Lichtkegels (Flamme einer Stearinkerze) nicht mehr erkennen liess. Daher geben die in nachstehender Tabelle unter der Rubrik "Galactoskop" verzeichneten Zahlen die Cubikcentimeter an, welche an Milch verbraucht sind, um die erwälnte Menge Wasser undurchsichtig zu machen.

Zur Controle dieser Galactoskop-Prüfungen wurden die festen Bestandtheile der Milch durch Eindampfen derselben im Wasserbade durch Wägung des Rückstandes, analytisch festgestellt.

Wie aus der Tabelle ersichtlich ist, liess ich es mir angelegen sein, die Milch von einer Reihe verschiedener Kühe unter ungleichen Futterverhältnissen zu prüfen und habe ich alle Umstände in Betracht gezogen, die in irgend einer Weise auf die Beschaffenheit der Milch influiren können. Dabei schien es mir namentlich von Wichtigkeit zu sein, die Minimalsumme der festen Bestandtheile der Milch zu bestimmen, welche bei zum Theil nahrungsarmem Stallfutter, also unter ungünstigen Verhältnissen, darin enthalten sein kann. Aus der Mannigfaltigkeit der Verhältnisse ergiebt sich klar die Nothwendigkeit einer längeren Versuchsreihe. Man wird darnach mit um so grösserer Sicherheit in Rücksicht auf die Menge der festen Bestandtheile eine scharfe Grenze feststellen können, über welche hinaus die Milch als verfälscht anzusehen ist.

In Betreff der Reihenfolge der Versuche habe ich in der Tabelle zuerst die Morgenmilch, dann die Mittagsmilch und schliesslich die Abendmilch aufgeführt. 


\begin{tabular}{|c|c|c|c|c|c|c|}
\hline 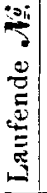 & $\begin{array}{l}\text { R a c e } \\
\text { der } \\
\mathrm{K} \text { u h. }\end{array}$ & $\begin{array}{c}\text { Alter } \\
\text { der } \\
\text { Kuh. } \\
\text { Jahre. }\end{array}$ & Futter der Kul. & $\begin{array}{l}\text { Wie oft } \\
\text { die } \\
\text { Kuh } \\
\text { ge- } \\
\text { kalbt. }\end{array}$ & \multicolumn{2}{|c|}{$\begin{array}{l}\text { Verflos. } \\
\text { sene Zeit } \\
\text { nach dem } \\
\text { Kalber. }\end{array}$} \\
\hline 1 & Hiesige. & 9 & $\begin{array}{l}\text { Küchenwäsche, Heu, Häcker- } \\
\text { ling, Küchenabfall. }\end{array}$ & $7 \mathrm{Mal}$ & $51 / 2$ & Monat \\
\hline 2 & do. & 8 & $\begin{array}{l}\text { Häckerling, Heu und Kartof- } \\
\text { feluabfall. }\end{array}$ & $6 n$ & 9 & $n$ \\
\hline 3 & do. & $6-7$ & $\begin{array}{l}\text { Heu, Klee, Stroh und Brannt- } \\
\text { weinwäsche. }\end{array}$ & $5 \%$ & 3 & 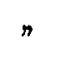 \\
\hline $\begin{array}{l}4 \\
5\end{array}$ & do. & $7 \stackrel{9}{-8}$ & $\begin{array}{l}\text { Runkelrïben, Häckerling, Kleie. } \\
\text { Fruchthülsen von der Stärke- } \\
\text { fabrik, Runkelrüben, Heu } \\
\text { und Häcksel. }\end{array}$ & 6 ?" & $\begin{array}{l}7 \\
9\end{array}$ & ” \\
\hline $\begin{array}{l}6 \\
7 \\
8\end{array}$ & $\begin{array}{l}\stackrel{?}{\text { Oldenburger. }} \\
\text { Hiesige. }\end{array}$ & $\begin{array}{r}5 \\
7 \\
11\end{array}$ & $\begin{array}{l}\text { Desgleichen. } \\
\text { Heu, Stroh, Seie u. Runkelrüben, } \\
\text { Heu, Haferstroh, Branntwein- } \\
\text { wäsche, Oelkuchen. }\end{array}$ & $\mid \begin{array}{ll}2 & n \\
5 & n \\
8 & n\end{array}$ & $\begin{array}{r}3 \\
3 \\
12\end{array}$ & 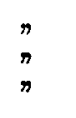 \\
\hline 9 & Ostfriesische. & 7 & Runkelrüben, Häckerling, Kleie, & 5 & 2 & $n$ \\
\hline $\begin{array}{l}10 \\
11\end{array}$ & $\begin{array}{l}\text { Oldenburger. } \\
\text { Ostfriesische. }\end{array}$ & $?$ & $\begin{array}{l}\text { Heu, Stroh, Runkelrüben, Seie. } \\
\text { Schrot, Oelkuchen, Heu, Hafer- }\end{array}$ & & $\begin{array}{l}4 \\
9\end{array}$ & $"$ \\
\hline 12 & Hiesige. & 11 & $\begin{array}{l}\text { Strohhäckerling, Runkeln, Schrot, } \\
\text { Malzkeime. }\end{array}$ & $8 n$ & 8 & 》 \\
\hline $\begin{array}{l}13 \\
14\end{array}$ & $\begin{array}{l}\text { Ostfriesische. } \\
\text { do. }\end{array}$ & $\begin{array}{l}6 \\
7\end{array}$ & $\begin{array}{l}\text { Runkelrüben, Häckerling, Kleie. } \\
\text { Desgleichen. }\end{array}$ & $\begin{array}{ll}4 \\
5\end{array}$ & $\begin{array}{l}4 \\
8\end{array}$ & $\pi$ \\
\hline 15 & Haiderace. & 7 & $\begin{array}{l}\text { Branntwein wäsche, Seie, Häcker- } \\
\text { ling, Heu. }\end{array}$ & & $10 \% / 2$ & $n$ \\
\hline 16 & Hiesige. & 12 & Stroh. & ? & 6 & n \\
\hline 17 & do. & $6-7$ & $\begin{array}{l}\text { Heu, Klee, Stroh und Brannt- } \\
\text { weinwäsche. }\end{array}$ & & 3 & $n$ \\
\hline 18 & Oldenburger. & 8 & $\begin{array}{l}\text { Stroh, Heu, Seie und Runkel- } \\
\text { rüben. }\end{array}$ & 6 & 6 & $n$ \\
\hline 19 & Hiesige. & 8 & $\begin{array}{l}\text { Häckerling, Heu, Kartoffeln- } \\
\text { abfall. }\end{array}$ & $6 \%$ & 10 & $n$ \\
\hline \begin{tabular}{|}
20 \\
21
\end{tabular} \mid & $\begin{array}{l}\text { Oldenburger. } \\
?\end{array}$ & $7 \stackrel{7}{-8}$ & $\begin{array}{l}\text { Seie, Heu, Stroh u. Runkelrüben. } \\
\text { Fruchthülsen von der Stärke- } \\
\text { fabrik, Runkelrüben, Heu } \\
\text { und Häcksel. }\end{array}$ & $5 ?$ & $\begin{array}{l}3 \\
9\end{array}$ & $"$ \\
\hline $\begin{array}{l}22 \\
23\end{array}$ & $\stackrel{?}{\text { Hiesig }}$ & $\begin{array}{l}5 \\
\mathbf{9}\end{array}$ & $\begin{array}{l}\text { Desgleichen. } \\
\text { Runkelrüben, Häckerling, Kleie. }\end{array}$ & $\begin{array}{ll}2 & n \\
6 & n\end{array}$ & $\hat{7}$ & $n$ \\
\hline 24 & Oldenburger. & 7 & $\begin{array}{l}\text { Heu, Stroh, Seie und Runkel- } \\
\text { ruben. }\end{array}$ & $5 \%$ & 3 & $n$ \\
\hline 25 & Hiesige. & 7 & $\begin{array}{l}\text { Heu, Strohbäckerling, Runkel- } \\
\text { rüben. }\end{array}$ & $5 n$ & 10 & $\pi$ \\
\hline 26 & Ostfriesische. & $\left.21\right|_{2}$ & Seie, Stroh, Heu und Runkel- & $1 \%$ & $51 / 2$ & ～ \\
\hline 27 & Hiesige. & 4 & $\begin{array}{l}\text { Hafer, Roggenschrot, Heu, Run. } \\
\text { keln und Strobbäckerling. }\end{array}$ & $1 n$ & 6 & 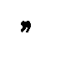 \\
\hline
\end{tabular}




\begin{tabular}{|c|c|c|c|c|}
\hline Melkzeit. & $\begin{array}{c}\text { Feste } \\
\text { Bestand. } \\
\text { theile } \\
\text { der Milch } \\
\text { in } 100 \\
\text { Theilen. }\end{array}$ & $\begin{array}{r}\text { Galac } \\
\text { skor }\end{array}$ & $\begin{array}{l}\text { cto- } \\
\text { p. }\end{array}$ & Bemerkungen. \\
\hline Morgens & 13,50 & $3,5 \mathrm{C}$ & C.C. & \\
\hline$\eta$ & 11,25 & 4,3 & & \\
\hline$r$ & 11,81 & 6,3 & $\eta$ & \\
\hline " & $\begin{array}{l}11,84 \\
11,44\end{array}$ & $\begin{array}{l}4,6 \\
6,0\end{array}$ & ” & Die Kuh wird zum Ziehen benutzt. \\
\hline$\dddot{7}$ & $\begin{array}{l}12,46 \\
12,43 \\
11,67\end{array}$ & $\begin{array}{l}3,5 \\
4,6 \\
4,4\end{array}$ & $\begin{array}{l}n \\
n\end{array}$ & Desgleichen. \\
\hline 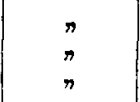 & $\begin{array}{l}11,46 \\
11,55 \\
13,14\end{array}$ & $\begin{array}{l}4,5 \\
6,3 \\
4,4\end{array}$ & $"$ & Die Kuh giebt sehr viel Milch. \\
\hline$\pi$ & 12,76 & 4,1 & $n$ & \\
\hline 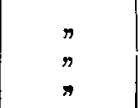 & $\begin{array}{l}11,76 \\
12,33 \\
11,81\end{array}$ & $\begin{array}{l}5,1 \\
3,8 \\
4,8\end{array}$ & $\stackrel{n}{n}$ & \\
\hline$\pi$ & 11,27 & 4,0 & $"$ & Die Kuh ist sehr mager und giebt nur \\
\hline Mittags & 13,20 & 4,0 & $"$ & Morgenmilch dieser Kuh siehe No. 3 . \\
\hline 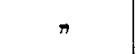 & 12,50 & 4,0 & » & \\
\hline$n$ & 12,50 & 4,3 & 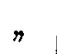 & \\
\hline 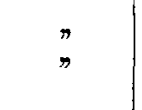 & $\begin{array}{l}11,32 \\
12,70\end{array}$ & $\begin{array}{l}4,6 \\
3,7\end{array}$ & 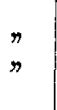 & $\begin{array}{l}\text { Die Morgenmilch dieser Kuh siehe No. } 7 \text {. } \\
\text { Die Kuh wird zum Ziehen benutzt; Morgen } \\
\text { milch dieser Kuh siebe No. } 5 \text {. }\end{array}$ \\
\hline$n$ & 12,23 & $\begin{array}{l}2,9 \\
2,7\end{array}$ & $n$ & $\begin{array}{l}\text { Desgl.; Morgenmilch dieser Kuh siehe No. } 6 . \\
\text { Morgenmilch dieser Kub siehe No.4. }\end{array}$ \\
\hline$\pi$ & 11,10 & 5,5 & $"$ & $n \quad \pi \quad n \pi 7$ \\
\hline$\pi$ & 13,05 & 4,4 & $\eta$ & \\
\hline 》 & 13,20 & 3,8 & $\eta$ & \\
\hline$\pi$ & 11,83 & 4,9 & $n$ & \\
\hline
\end{tabular}




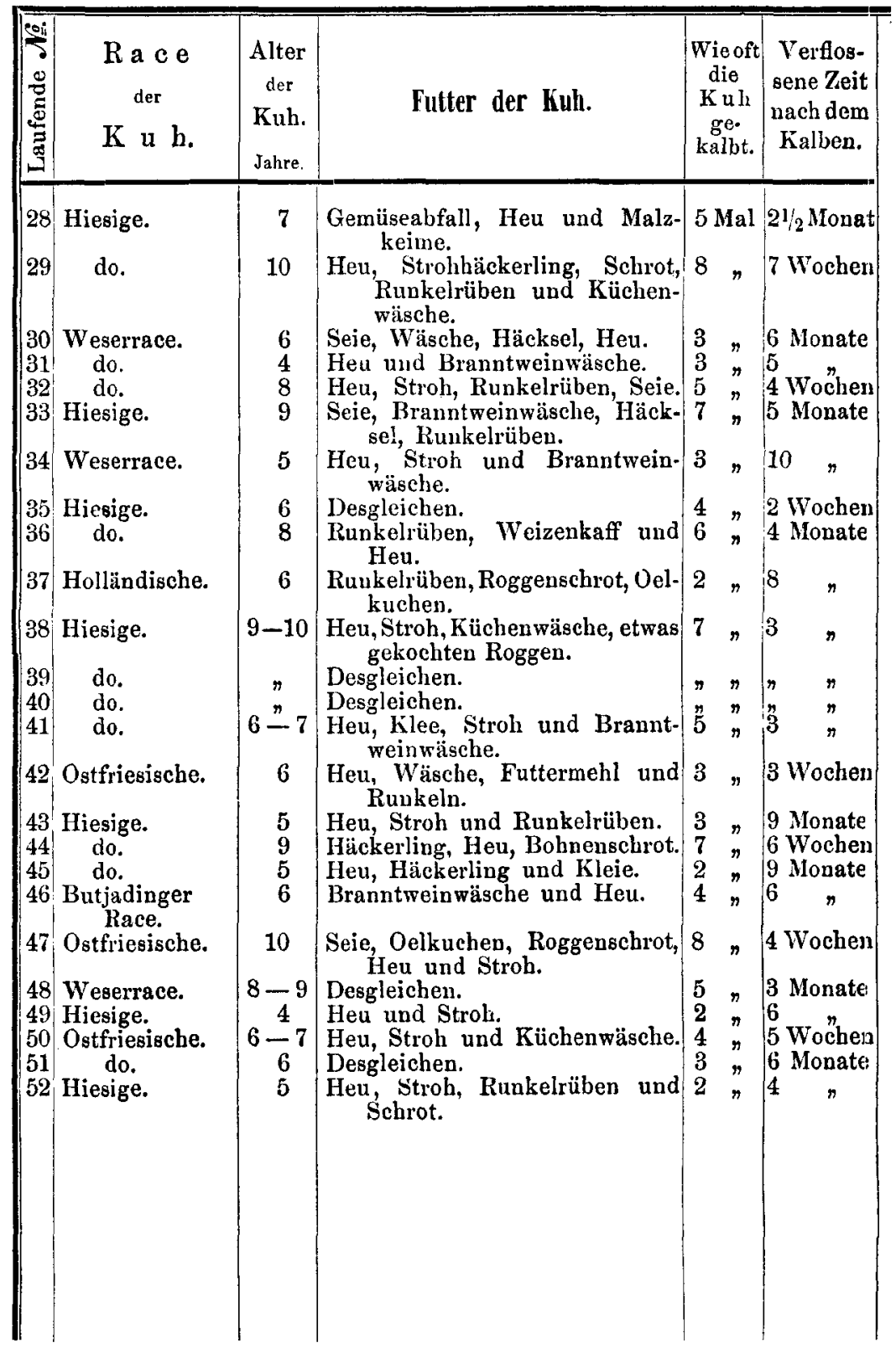




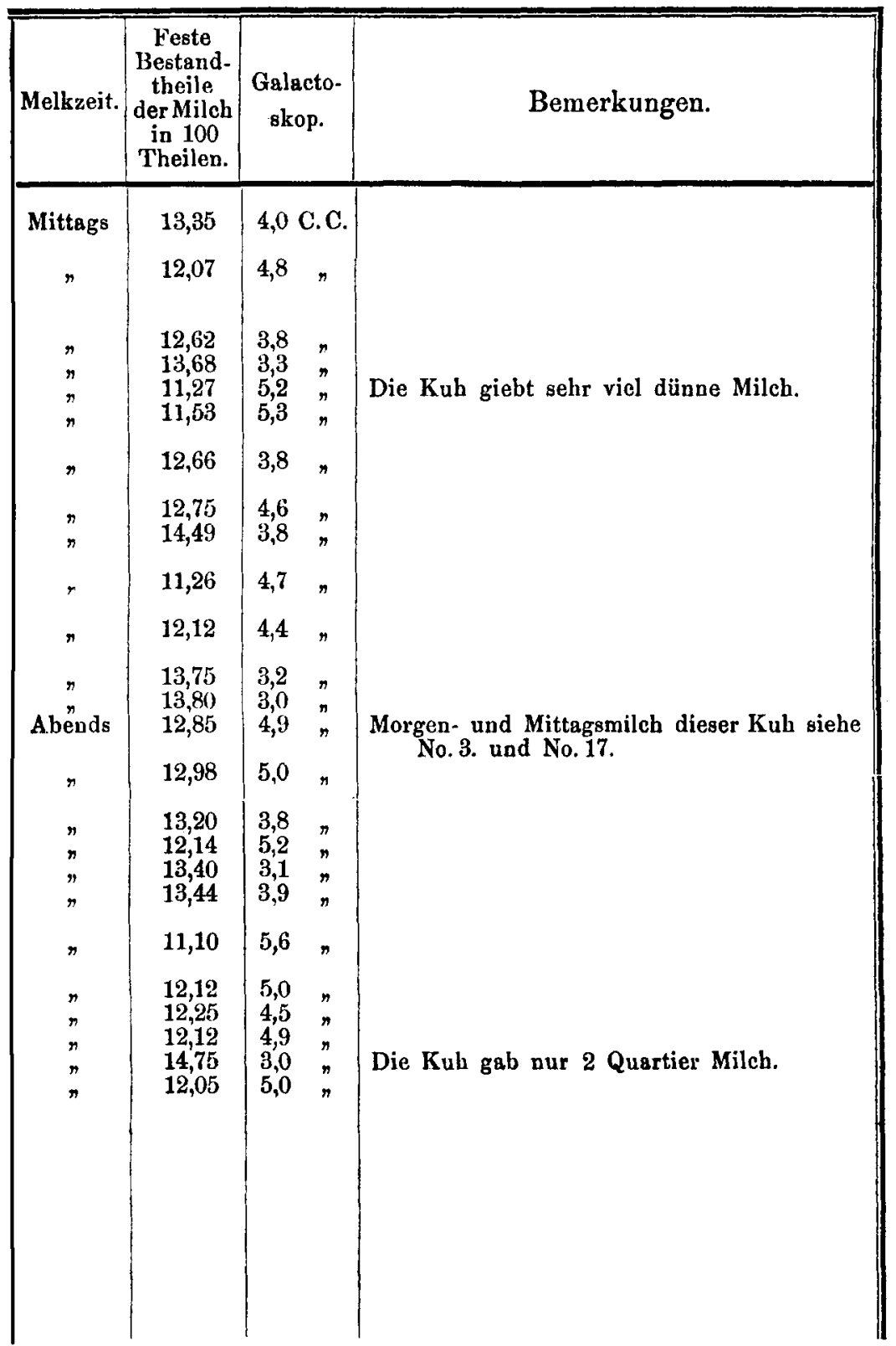

Arch. d. Pharm. CLXXXII. Bds, 3. Hft. 
Betrachten wir das Ergebniss der 52 Versuche, so variiren die festen Bestandtheile von 11,10 Procent bis 14,75 Procent, jedoch sind diese Verschiedenheiten nicht so gross, als sie auf den ersten Blick sich darstellen, wenn man die Versuche classificirt. Von 11,10-11,25 Proc. sind bei 52 Versuchen nur zwei Fälle vorgekommen, dagegen von 11,25-13,80 Proc. 48, während über 13,80 Proc. ebenfalls nur zwei Fälle vorkamen.

Hieraus leuchtet ein, dass die Grenzen der festen Bestandtheile der Normal-Milch regelmässig 11,25 und 13,80 Proc. sind, mithin nur eine Schwankung von ungefähr $2^{1 / 2}$ Proc. zulassen. Mit Rücksicht auf die vorhin erwähnten beiden, allerdings sehr vereinzelt dastehenden Fälle von 11,10 Proc. kann ich jedoch erst dann eine Milch als verfälscht ansehen, wenn die Untersuchung nur 11 Proc. feste Bestandtheile oder darunter angiebt. Als Hauptargument für die Annahme von 11,00 Proc. spricht namentlich der Versuch No. 16. Die Milch war von einer alten, sehr magern Kuh, die nur mit Stroh gefüttert wurde, entnommen und dennoch enthielt sie 11,27 Proc. feste Bestandtheile.

Haben wir nun eine Milch, die nur 11 Proc. feste Bestandtheile oder darunter enthält, also eine mit Wasser verfälschte, so berechnet sich die Menge des fälschlich beigemischten Wassers nach der aus den 52 Versuchen hervorgehenden Mittelzahl, da man hierbei die selten vorkommenden Minimal- und Maximal-Summen offenbar nicht zu Grunde legen darf.

Es wurden im Mittel sämmtlicher Versuche 12,44 Proc. feste Bestandtheile gefunden und kann man in runder Zahl 12 Proc. feste Bestandtheile für die Berechnung des Wassergehaltes als Norm annehmen. Die GalactoskopPrüfungen schwanken von 2,7-6,3 C.C. Für diese Variationen gilt dasselbe, was vorher von den festen Bestandtheilen gesagt ist, nämlich dass regelmässig nur 3,0 bis 5,5 C.C. Milch verbraucht werden, jedoch darüber und darunter innerhalb der Grenzen 2,7 und 6,3 C.C. vereinzelte Fälle vorkommen. 
Vergleichen wir nun die optischen Proben mit den gewichtsanalytischen, so sieht man auf den ersten Blick, dass zwischen jenen und diesen keine mathematischen Relationen festzustellen sind, wenngleich im Allgemeinen die nach der Bestimmung der festen Bestandtheile schlecht sich erweisende Milch auch durch die optische Probe mit dem Galactoskop als solche erkannt wurde. Es war vorauszusehen, dass ein Instrument, welches nur auf den Fettgehalt der Milch begründet ist, also nur einen, wenngleich einen der vorzüglichsten der festen Bestandtheile beurtheilen lässt, nicht genau mit der gewichtsanalytischen Prüfung stimmen kann, welche auf eine Berücksichtigung der sämmtlichen Bestandtheile basirt. Zum Beispiele ist bei No. 4 und 7 der Fettgehalt vollständig gleich, es wurden 4,6 C.C. Milch zur optischen Probe verwandt, dagegen differiren die festen Bestandtheile; denn No. 4 hat 11,84 Proc. und No. $7=12,43$ Proc. Ferner sind bei No. 16 und 32 die Summen der festen Bestandtheile gleich, in beiden Fällen wurden genau 11,27 Proc. gefunden. Die Galactoskop-Prüfung ergab bei No. $32=5,2$ C.C. und bei No. $16=4,0$ C.C., also war der Rahmgehalt verschieden.

Es fragt sich nun, ob bei dieser allerdings nicht bedeutenden Inconvenienz das Galactoskop seine Aufgabe als praktisches Voruntersuchungs-Instrument noch erfüllen kann. Ich werde diese Frage unten bei den Versuchen aus der Praxis erörtern.

Ein Umstand, der schon früher von andern Chemikern beobacbtet worden, ist mir auch aufgefallen, nämlich, dass die zuerst aus dem Euter fliessende Milch fettärmer ist, als die später ausfliessende. Durch die Versuche No. 38,39 und 40 wurde dieses experimental bestätigt. Die zuerst aufgefangene Milch (No. 38) ergab 4,4 C.C. nach dem Galactoskop, während die mittlere Milch (No. 39), wie aus den optischen Daten zu ersehen ist, mehr Fett enthielt und die zuletzt ausfliessende (No. 40) mit der mittleren fast gleichen Buttergehalt hatte. Auch habe 
ich bestätigt gefunden, was andere Beobachter angeben, dass im Allgemeinen die Morgenmilch schlechter ist, als die Mittagsmilch und die letztere nicht so gut als die Abendmilch. Aus den Analysen der Morgenmilch berechnen sich im Mittel 12,03 Proc. feste Bestandthcile, aus denen der Mittagsmilch 12,60 Proc. und die Abendmilch ergab im Mittel 12,70 Proc.

Der Umstand, dass die Morgenmilch schlechter zu sein pflegt, als die Mittagsmilch und Abendmilch, so wie ferner, dass frischmelkende Kühe im Allgemeinen dünnere Milch liefern, als altmilchende ist noch nicht hinreichend erklärt. Ich glaube jedoch, dass jene Erscheinung darin ihren Grund hat, dass in den gedachten Füllen grössere Mengen Milch secernirt werden, als sonst, dass also die Milch um so geringerer Qualität ist, je grössere Quantitäten Milch die Kuh giebt.

\section{Versuche aus der Pracis.}

Nachdem die Arbeiten mit Normalmilch beendet waren, schien es mir ein Haupterforderniss, zu der Praxis überzugehen, da hier ganz andere Verhältnisse in den Vordergrund treten. Die im Handel rorkommende Milch ist meistens nicht von einer Kuh, es ist ein Gemisch von verschiedenen Milchsorten und ist dieselbe den mannigfaltigsten Behandlungen ausgesetzt. Theils wird die Milch mehr oder weniger abgerahmt, theils mit Wasser verdünnt und häufig geschieht sogar Beides. Wird mit der gemischten Normalmilch des Handels nichts vorgenommen, so ist leicht einzusehen, dass diese von mehren Kühen stammende Milch in Hinsicht ihres Procentgehaltes an festen Bestandtheilen dem oben für sämmtliche Versuche angegebenen mittleren Procentgehalt sich nähert; denn man kann nach allen Erfahrungen nicht annehmen, dass eine ganze Reihe von Kühen nur sehr schlechte oder nur sehr gute Milch gebe.

$\mathrm{Zu}$ den Versuchen benutzte ich die Milch, wie sie den Consumenten feilgeboten wurde. Die Resultate der Analysen ordnete ich in zwei Tabellen; die eine bezieht 
sich auf die Milch, welche als unverfälscht befunden wurde, die andere enthält die Untersuchungen der verfälschten Milch. Beide Tabellen sind von grosser Wichtigkeit. Die Tabelle der guten Milch wird zeigen, wie das Einschreiten der betreffenden Behörde auf die Beschaffenheit der Handelsmilch eingewirkt hat. Die Tabelle der schlechten Milch ist einerseits ein Prüfstein für das Galactoskop, andererseits verbreitet sie Licht über die Art und Weise der Milchfälschung.

\begin{tabular}{|c|c|c|c|c|c|c|c|c|}
\hline \multicolumn{5}{|c|}{ Schlechte Milch. } & \multicolumn{4}{|c|}{ Gute Milch. } \\
\hline 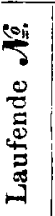 & $\begin{array}{c}\text { Feste } \\
\text { Bestand. } \\
\text { theile } \\
\text { der Milch } \\
\text { in } \\
100 \text { Theilen. }\end{array}$ & \multicolumn{2}{|c|}{$\begin{array}{c}\text { Galacto- } \\
\text { skop. }\end{array}$} & $\begin{array}{c}\text { Abuormer } \\
\text { Wasser- } \\
\text { gehalt nach } \\
12 \% \text { festen } \\
\text { Bestand- } \\
\text { theilen } \\
\text { berechnet. }\end{array}$ & 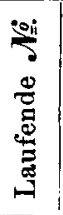 & $\begin{array}{c}\text { Feste } \\
\text { Bestand- } \\
\text { thejle } \\
\text { der Milch } \\
\text { in } \\
100 \text { Theilen. }\end{array}$ & \multicolumn{2}{|c|}{$\begin{array}{c}\text { Galacto- } \\
\text { skop. }\end{array}$} \\
\hline 1 & 9,28 & 17,0 & C.C. & 22,67 & 1 & 12,44 & 3,6 & C.C. \\
\hline 2 & 9,72 & 7,0 & 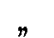 & 19,00 & 2 & 11,25 & 5,5 & \\
\hline 3 & 10,00 & 21,0 & & 16,67 & 3 & 11,50 & 6,2 & \\
\hline 4 & 10,23 & 10,0 & & 14,75 & 4 & 11,88 & 5,5 & \\
\hline 5 & 8,87 & 7,5 & & 16,25 & 5 & 12,83 & 4,9 & \\
\hline 6 & 10,90 & 6,5 & $n$ & 9,17 & 6 & 11,27 & 7,5 & $\pi$ \\
\hline 7 & 10,90 & 6.2 & & 9,17 & 7 & 11,97 & 4,9 & $\eta$ \\
\hline 8 & 10,82 & 6,0 & $n$ & 9,84 & 8 & 12,50 & 4,8 & 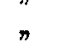 \\
\hline 9 & 10,00 & 7,0 & $n$ & 16,67 & 9 & 11,35 & 5,5 & $\pi$ \\
\hline 10 & 5,88 & 9.7 & 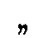 & 51,00 & 10 & 11,93 & 4,8 & n \\
\hline 11 & 11,00 & 6,5 & $n$ & 8,34 & 11 & 14,60 & 2,5 & $\pi$ \\
\hline 12 & 9,50 & 6,1 & $n$ & 20,84 & 12 & 11,25 & 6,4 & $\pi$ \\
\hline 13 & 11,00 & 6,7 & $n$ & 8,34 & 13 & 12,10 & 4,9 & $\pi$ \\
\hline 14 & 10.90 & 6,3 & $n$ & 9.17 & 14 & 12,00 & 4,3 & $\pi$ \\
\hline 15 & 9,87 & 7,4 & $n$ & 17,75 & 15 & 11,11 & 5,4 & $n$ \\
\hline 16 & 10,25 & 7,9 & $n$ & 14,59 & 16 & 11,80 & 5,3 & $n$ \\
\hline 17 & 10,25 & 6,5 & $n$ & 14,59 & 17 & 11,80 & 4,3 & $n$ \\
\hline 18 & 10,35 & 7,4 & 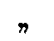 & 13,75 & 18 & 13,20 & 3,8 & $n$ \\
\hline 19 & 10,96 & 5.8 & $n$ & 8,67 & 19 & 12,25 & 4,5 & $n$ \\
\hline 20 & 11,00 & 6,0 & $n$ & 8,34 & 20 & 12,02 & 4,8 & $n$ \\
\hline 21 & 10,60 & 5,9 & $n$ & 10,67 & 21 & 11,23 & 5,8 & $\eta$ \\
\hline 22 & 9,92 & 5,8 & $n$ & 17,34 & 22 & 12,10 & 5,7 & $\pi$ \\
\hline 23 & 10,69 & 5,9 & $n$ & 10,92 & 23 & 12,10 & 5,8 & $n$ \\
\hline & & & & & 24 & 11,49 & 5,2 & $n$ \\
\hline & & & & & 25 & 12,54 & 5,8 & $n$ \\
\hline
\end{tabular}


Kritisiren wir zunächst die Resultate der Tabelle über schlechte Milch, so zeigt Versuch No. 1. eine Milch, die abgerahmt und mit Wasser verdünnt ist. No. 2. eine Milch, die mit Wasser verdünnt ist. No. 3. eine Milch, die sehr stark abgerahmt ist, ohne Wasserzusatz. No. 10 eine Milch, die sehr mit Wasser verdünnt ist, ohne abgerahmt zu sein. Die übrigen Milchsorten sind sämmtlich mit Wasser vermischt, oder schwach abgerahmt. Die Frage, ob die Fälschung durch Wasserzusatz oder durch Abrahmen, oder durch Beides zusammen entstanden, lässt sich mit einiger Wahrscheinlichkeit nur in den ganz eclatanten, Fällen beantworten, in den anderen meistens vorkommenden Fällen aber ist weder durch die optische noch durch die gewichtsanalytische Prüfung auch nur annähernd sicher jene Frage zu entscheiden. Soll die Möglichkeit der Annahme, dass die Milch lediglich abgerahmt sei, gänzlich ausgeschlossen werden, so muss die Norm der festen Bestandtheile so sehr heruntergesetzt werden, dass die Milchcontrole vollständig ihre Bedeutung verliert. Nach meiner Ansicht dürfte aber jener Frage eine Wichtigkeit überall nicht beizumessen sein; denn in jedem dieser Fälle sind mit der Milch, wie sie die Natur liefert, durch positive Handlungen Veränderungen zum Nachtheile des Publicums vorgenommen, sei es nun durch directe Entziehung der festen Bestandtheile, sei es durch Verminderung derselben in Folge Wasserzusatzes. Der Zusatz von Wasser benachtheiligt das Publicum doch offenbar nur insofern, als dadurch die festen Bestandtheile der Milch verringert und in Folge dessen die Nahrungskraft der letzteren vermindert wird.

Ganz und gar dasselbe aber findet Statt bei einer directen Entziehung der nahrungskräftigen Bestandtheile durch Abrabmen.

Wenn daher das Strafwürdige bei der Versetzung der Milch mit Wasser darin liegt, dass der Nahrungsgehalt und Werth zum Schaden der Consumenten 
verringert wird, so folgt daraus mit nothwendiger Consequenz die Strafbarkeit des Abrahmens der Milch. Das Eine steht und fällt mit dem Anderen. Sehen sich die Verkäufer genöthigt, oder ist es ihr Wille, den Consumenten abgerahmte Milch feilzubieten, so müssen sie dieselbe als solche bezeichnen und billiger verkaufen.

Die Tabelle über die gute Milch giebt einen schlagenden Beweis, dass das Einschreiten der Behörde den Milchconsumenten gute Dienste geleistet hat. Unter 48 Milchsorten waren 25 Sorten unverfälscht und ist unter diesen unverfälschten Milchsorten zum grössten Theil sehr gute Milch und nur ein kleiner Theil derselben steht der für die schlechte Milch angenommenen Grenze $(11,00)$ nahe.

Erörtern wir nun im Folgenden die Frage, ob die optische Milchprüfung der praktischen Voruntersuchung die gewünschten und nöthigen Dienste leisten kann.

Aus der Tabelle der Normalmilch haben wir ersehen, dass die optischen Prüfungen von $2,7-6,3$ C.C. differiren und finden, dass sich fast dasselbe Resultat bei der guten Milch in der Praxis herausstellt. Die Variationen sind hier, abgesehen von einer Ausnahme (No. 6), von 2,5-6,4 C.C.

Betrachten wir nun die optischen Proben der schlechten Milch, so ergiebt sich das sehr befriedigende Resultat, dass keine Milchsorte unter 5,8 C.C. zur optischen Prüfung verbrauchte; denn wenn bei Untersuchungen der schlechten Milch weniger Cubikcentimeter hinreichend gewesen wären, um den Lichtkegel verschwinden zu machen, so würden diese Prüfungen mit den optischen Proben der Normalmilch in eine derartige Collision gerathen, dass das Galactoskop völlig unbrauchbar wäre. Eine kleine Collision zwischen Normalund abnormer Milch ist natürlich bei der eben erklärten Einseitigkeit des Instrumentes nicht zu vermeiden, jedoch bieten die leichte Handhabung und schnelle Ausführung 
der optischen Prüfung so grosse Vortheile dar, dass jener Uebelstand dagegen verschwindet.

Wollen wir nun das Galactoskop so scharf einstellen, dass unserer Ueberzeugung nach so leicht kein Milchfälscher nach der angenommenen Norm der festen Bestandtheile ungestraft bleibt, so ist es klar, dass einige Sorten Milch, wie aus den Resultaten der Tabelle hervorgeht, durch das optische Instrument für schlecht befunden werden, die nach der Gewichtsanalyse noch gut sind. Jedenfalls ist es besser, dass Analysen vergebens gemacht werden, als dass man bei der GalactoskopPrüfung eine Grenze annimmt, die einen Theil der fălschenden Milchverkäufer ungestraft lässt.

Nach reiflicher Ueberlegung, mich stützend auf die Ergebnisse der Tabellen, halte ich es für das Beste, anzunehmen, dass die Milch bei der Voruntersuchung dann als verfälscht anzusehen ist, wenn 100 C. C. Wasser mit 5,5 C.C. Milch versetzt werden und die Conturen des Lichtkegels noch zu sehen sind. Wird hierbei gen a u beobachtet, so wird eine Fälschung der Milch schwerlich unentdeckt bleiben und bliebe nur die Mühe einiger vergeblichen Analysen, die man im Interesse des Publicums gern ausführen kann, wenn eben dadurch das angestrebte Ziel erreicht wird, dass ein so wichtiges Nahrungsmittel, wie die Milch, dem Publicum unverfälscht verkauft wird.

Hannover, im Juli 1867. 